\title{
Análise comparativa entre modelos de estoque
}

\author{
Miguel Cezar Santoro
}

Gilberto Freire

EPUSP

\begin{abstract}
Resumo
Este trabalho é um estudo do problema de escolha entre modelos de estoque para a gestão de itens independentes em local único. Quatro modelos de estoque são considerados, sendo três deles reativos, que não utilizam previsão de demanda para a tomada de decisões, e um ativo, que decide com base em previsões. A medida de desempenho considerada é o custo de operação médio por período, que é obtida através de simulação da utilização dos modelos com base num histórico de vendas. 0 trabalho analisa o efeito da alteração de previsibilidade e da consideração de quantidades mínimas de compra no modelo ativo no desempenho relativo dos modelos analisados. A análise utiliza um modelo de busca acoplado a um simulador. 0 trabalho apresenta subsídios para a escolha e parametrização de modelos de estoque em empresas.
\end{abstract}

Palavras-chave

Modelos de estoques, simulação, busca.

\section{Inventory models comparative analysis}

\begin{abstract}
This paper is an approach of the inventory models selection problem in single echelon system management with independent items. Four inventory models are considered, three reactive, which don't use demand forecasting for decision making, and one active model, that decides based on forecasting. Average operation cost per period is the performance measurement variable considered, calculated by using simulation of the models applied on sales temporal series. The paper analyses the effects, in the relative models performance, of the demand forecast accuracy and the use of minimum acquisition quantity in the active model. The analysis uses a search model jointly a simulator to optimize the parameters. The results present useful information for models selection and parameters determination in organizations environment.
\end{abstract}

Key words

linventory models, simulation, search. 


\section{INTRODUĈ̣̃O}

O problema de estoques há muito tempo pode utilizar modelos matemáticos como auxílio à sua aplicação, tendo sido por volta de 1915 a primeira publicação da conhecida fórmula do lote econômico desenvolvida por F. W. Harris. A abordagem matemática do problema iniciou-se efetivamente durante a $2^{\underline{a}}$ Guerra Mundial, e enfatiza a formulação de modelos ou políticas para a determinação do ponto de pedido (instante) em que devem ser efetuados os pedidos e da quantidade do mesmo (lote) sob determinadas propriedades e restrições da demanda, da oferta e dos custos do sistema considerado.

Citam-se como obras importantes para a disseminação dessa abordagem os artigos de Dvoretzky e Kiefer (1952), de Arrow; Harris e Marschak (1958) e os livros de Hadley e Whitin (1963), Naddor (1966), e Brown (1967 e 1977). para atender as necessidades. Essa forma de compra é conhecida como Lote a Lote, embora seja questionável tal nome. Esses modelos e suas variações, daqui por diante, serão denominados Cálculo de Necessidades, para diferenciá-los dos MRP, que cuidam de processos de transformação com demandas dependentes.

Apesar da idéia conceitual e original do Cálculo de Necessidades ser a aquisição do estritamente necessário, surge naturalmente o problema de altos custos de aquisição quando existirem custos de pedido altos. Uma série de modelos de agrupamento em lotes foi criada, então, para resolver esse problema. Eles podem ser vistos, por exemplo, em Hax e Candea (1984) e Orlicky (1995).

Essa abordagem ativa (aquisições determinadas por previsões) provocou discussões sobre o acoplamento de modelos de previsão ao de estoques. Podemos ver em Fildes e Beard (1992) uma discussão interessante sobre medidas de previsibilidade e em Gardner (1990) uma análise de como a qualidade das previsões pode afetar as decisões e o custo delas num sistema de estoques.

O aumento da complexidade dos problemas surgidos com os modelos ativos, por outro lado, favoreceu a

Os primeiros modelos disponibilizados por essa abordagem, hoje denominados tradicionais ou reativos, são modelos que permitem tomar as decisões de quando e quanto abastecer sem que seja necessário obter previsões sobre a demanda. A demanda, na absoluta maioria dos estudos, é considerada contínua, com média constante e explicada por uma distribuição de probabilidade fixa e conhecida no tempo.

Na década de 1970, Eilon e Elmaleh (1970) apresentaram um modelo que utiliza previsões para ajuste adaptativo de parâmetros dos modelos reativos de estoque, enquanto que, paralelamente, discutia-se nos meios empresariais e acadêmicos a filosofia Just in Time (JIT), e o conseqüente interesse na compra do estritamente necessário, e não de lotes. Surgiram então os chamados sistemas Material Requirement Planning (MRP). Eles serviram, inicialmente, para tratar decisões de abastecimento em problemas de produção (estoques intermediários) de itens com demandas dependentes (não comercializados diretamente), que apareciam nas estruturas de fabricação e montagem de produtos com demandas independentes (comercializados). O cronograma aproximado e pontos importantes do desenvolvimento desses e de outros modelos de estoque e de programação podem ser vistos em Sipper e Bulfin. (1998).

Como conseqüência de considerar demandas com tendências, sazonalidades e saltos, e a filosofia JIT, naturalmente surgiram decisões de compra baseadas diretamente em previsões, nas quais se compra o estritamente necessário utilização da simulação e de modelos de busca como instrumentos exploratórios e de tomada de decisões próximas às ótimas nesse novo ambiente. Exemplos de uso interessante dessas ferramentas podem ser vistos em Fu e Healy (1997) e Lopez-Garcia e Posada-Bolivar (1999).

O foco deste estudo é efetuar uma análise comparativa do desempenho entre modelos de estoque reativos e ativos, quando submetidos a demandas com diferentes previsibilidades. O modelo ativo Cálculo de Necessidades é apresentado em duas versões, uma obedecendo aos preceitos da abordagem JIT, não formando lotes, e outra com a permissão de formação de lotes obedecendo a uma quantidade mínima. A consideração de quantidade mínima e não de lote permite a consideração de demandas altas, uma vez que não existe limitação superior.

A idéia básica é verificar se o comportamento do modelo Cálculo de Necessidades supera os reativos mesmo com baixa previsibilidade de demanda e se, para o caso de operação em ambientes com custos de pedido altos, onde trabalha com desvantagem pela não formação de lotes, a opção de quantidades mínimas de aquisição permite superar os modelos reativos. Considera-se essa avaliação como muito importante para a efetiva utilização de modelos ativos no ambiente das empresas.

Na próxima seção, o problema estudado é caracterizado, bem como são detalhados os objetivos do estudo. Seguindo, é apresentada a modelagem do problema e descrição dos 
dados de entrada, do tratamento efetuado e dos dados de saída. Na Seção "Resultados computacionais", os resultados obtidos são analisados. Na última seção, são apresentadas as principais conclusões do trabalho.

\section{DESCRIC̣ÃO DO PROBLEMA}

O problema objeto do estudo é o de itens sem estrutura estocados em local único, sujeitos a demandas regulares, que obedecem a quatro curvas de demandas médias: reta constante, reta inclinada, reta constante com sazonalidade e reta inclinada com sazonalidade. A oferta é ilimitada com tempo de espera constante, e a disponibilidade de recursos para armazenagem é também ilimitada. Os custos de pedidos, estocagens e faltas são fixos e conhecidos. Faltas convertem-se em atrasos.

Para a previsão será utilizado o modelo de projeção com reta inclinada e sazonalidade, e suavização exponencial simples nos três coeficientes de suavização.

Serão considerados quatro modelos de estoque, todos periódicos e com período de revisão unitário, sendo três deles reativos (Reposição do Máximo, Reposição da Base e Lote Fixo) e um ativo (Cálculo de Necessidades). A avaliação de desempenho dos modelos de estoque para cada item será feita pela simulação dos mesmos aplicados ao item num horizonte de 60 períodos, e pela medição do custo de operação médio por período, obtido pela soma dos custos de pedidos, armazenagens e faltas médios por período. Por facilidade de explicação, daqui por diante consideraremos a unidade de tempo mês.

Os objetivos do trabalho são:

- Efetuar uma análise do comportamento relativo dos modelos ativos e reativos em função de diferentes previsibilidades do modelo ativo.

- Analisar o efeito da introdução do parâmetro Necessidade Líquida Mínima no modelo ativo, no comportamento relativo já estudado.

\section{MODELAGEM DO PROBLEMA}

Nesta seção apresentamos o procedimento experimental, que se apóia em simulação e busca nos itens de estoque.

\section{trabalho analisa o efeito da alteração de previsibilidade e de quantidades mínimas de compra no desempenho dos modelos analisados.}

\section{Descrição dos Dados de Entrada}

A simulação foi efetuada com itens agrupados em famílias. Cada família foi caracterizada por uma curva de demanda, por um desvio-padrão e pelo tempo de espera (lead time de suprimento) dos seus itens. Os valores de demanda de cada família foram gerados a partir de sorteio aleatório em curvas normais, centradas nos pontos da curva de demanda e utilizando o desvio-padrão correspondente.

A Tabela 1, abaixo, ilustra a composição das famílias de itens.

A combinação de 4 curvas de demanda, 5 desvios padrão e 3 tempos de espera resulta em 60 famílias diferentes. Para cada família foram geradas 5 replicações com 60 períodos cada, o que resultou num total de 300 itens.

As curvas consideradas têm um valor da constante no instante zero igual a 100 e, quando for o caso, inclinação de 2 e índices de sazonalidade colocados na Tabela 2, a seguir:

Tabela 1: Caracterização das famílias de itens.

\begin{tabular}{|c|c|c|}
\hline \multicolumn{2}{|c|}{ FAMÍLIAS DE ITENS } & $\begin{array}{c}\text { TEMPOS DE ESPERA } \\
\text { (MÊS) }\end{array}$ \\
\hline CURVAS DE DEMANDA & $\begin{array}{c}\text { DESVIOS PADRÃO } \\
\text { (UNIDADE) }\end{array}$ & 1 \\
\hline Constante & 3 & 3 \\
\hline Inclinada & 7 & 6 \\
\hline Constante com Sazonalidade & 12 & \\
\hline Inclinada com Sazonalidade & 18 & \\
\hline
\end{tabular}


Os custos importantes para o estudo estão colocados a seguir (a unidade monetária é irrelevante por ser um estudo comparativo), e foram escolhidos de forma a gerar soluções equilibradas para as análises comparativas:

custo do item igual a 10 ;

custo de pedido igual a 20 e a 80 ;

taxa de armazenagem de 0,02 por mês;

custo de falta igual a 4 e a 10 .

pelo Cálculo de Necessidades na fase seguinte de simulação dos modelos de estoque.

Cálculo dos parâmetros e do desempenho dos modelos de estoque

Os quatro modelos de estoque considerados e sua operação estão explicados a seguir:

Modelo de Reposição do Máximo (RepMaxC)

Parâmetros: Ponto de Pedido (PtoPed) e Estoque Máximo (EstMax).

A cada final de período avalia-se o estoque físico mais o "a receber" e compara-se com o ponto de pedido. Se for superior nada se pede e se for inferior faz-se

\section{Descrição do tratamento dos dados de entrada de saída}

\section{Cálculo da equação de projeção}

Para cada um dos 300 itens com 60 meses de demanda foi ajustada uma curva de projeção considerando como período de inicialização 24 meses e período de validação os 36 meses restantes. Esse ajuste foi feito considerando como curva uma reta inclinada com sazonalidade, e escolha dos três parâmetros por suavização exponencial simples de acordo com Makridakis e Wheelright (1998). As simulações foram suportadas por uma busca direta na superfície de resposta para encontrar o melhor ajuste, com os coeficientes de suavização variando no intervalo [0,00 - 0,30], e com dois níveis de resolução, com passos respectivamente de 0,05 e 0,02 . A busca localiza o mínimo com o passo maior e reinicializa a partir da melhor solução com o passo inferior, tendo quatro critérios de parada, para dar segurança ao valor do mínimo encontrado:

Total de 800 iterações;

300 limites ou valores piores obtidos;

300 valores mínimos obtidos;

4 mínimos seguidos em direções e sentidos opostos.

Os resultados principais dessa fase são as quatro equações de projeção e os correspondentes melhores coeficientes de suavização para cada item. A melhor curva encontrada foi então transladada para a data zero e, com os melhores coeficientes de suavização, geraram-se as previsões utilizadas um pedido para a reposição do máximo. Esse modelo às vezes é chamado simplesmente de Modelo de Reposição Periódica.

\section{Modelo de Reposição da Base (RepBaseC)}

Parâmetros: Estoque Máximo (ou Base) e Necessidade Líquida Mínima (NLiqMin).

A cada final de período soma-se o estoque físico e o "a receber" e faz-se um pedido igual à diferença entre o Estoque Máximo e esta soma, caso não exista Necessidade Líquida Mínima. Caso exista, faz-se o pedido desta diferença somente se ela for superior à NLiqMin. Esse modelo é conhecido também com Modelo do Estoque Base.

\section{Modelo do Lote Fixo (LotFixC)}

Parâmetros: Ponto de Pedido (PtoPed) e Lote Fixo (LotFix). A cada final de período avalia-se o estoque físico mais o "a receber" e compara-se com o ponto de pedido. Se for superior nada se pede e se for inferior faz-se um pedido igual ao lote fixo. Esse modelo é conhecido também com Modelo do Lote Econômico quando o cálculo do lote obedece a alguma fórmula de lote econômico.

\section{Modelo do Cálculo de Necessidades (CalNec)}

Parâmetros: Estoque de Segurança (EstSeg) e Necessidade Líquida Mínima (NLiqMin).

A cada final de período avalia-se o estoque físico mais o "a receber" menos a demanda prevista no tempo de

Tabela 2: Îndices de sazonalidade.

\begin{tabular}{|c|c|c|c|c|c|c|c|c|c|c|c|}
\hline JAN & FEV & MAR & ABR & MAI & JUN & JUL & AGO & SET & OUT & NOV & DEZ \\
\hline 0,80 & 1,10 & 1,30 & 1,20 & 1,00 & 0,90 & 0,90 & 1,00 & 1,20 & 1,30 & 1,10 & 0,80 \\
\hline
\end{tabular}


reação mais o estoque de segurança. O tempo de reação é a soma do período de revisão mais o tempo de espera do item. Se essa quantidade for nula ou positiva nada se pede, e, se for negativa, faz-se um pedido igual à mesma, caso ela seja superior à NLiqMin. Caso seja igual ou inferior, pede-se a NLiqMin.
Não houve preocupação em melhorar os valores sementes, pois eles serviram, de fato, para a definição do passo e início da busca, mas não devem ter afetado o resultado da mesma, por se tratar de busca com três níveis de resolução, limites de variação dos parâmetros em relação às sementes e critérios de parada bastante frouxos.

Para cada um dos 300 itens com 60 meses de demanda e cada um dos três modelos de estoque é feita uma simulação da operação do modelo, iniciando-se na data zero e terminando-se no horizonte de simulação correspondente aos 60 meses.

\section{trabalho apresenta subsídios para a escolha e parametrização de modelos de estoque em empresas.}

Nessa simulação são medidos o número médio de pedidos/mês, o estoque médio/mês e as faltas médias/mês. Uma vez multiplicados pelos respectivos custos unitários de pedido, de estoque e de faltas e somados, resulta o custo médio de operação por período, que é o mensurador do desempenho do modelo de estoque para aquele item com os parâmetros simulados.

As simulações foram suportadas por uma busca direta com múltiplos reinícios na superfície de resposta para encontrar o melhor ajuste, com três níveis de resolução, com passos respectivamente de $0,06,0,02$ e 0,01 do valor semente do parâmetro. A busca localiza o mínimo com o passo maior e reinicializa a partir da melhor solução com o passo inferior, e assim por diante, tendo critérios de parada iguais ao da busca da projeção, para dar segurança ao valor do custo de operação mínimo encontrado. Os valores sementes dos parâmetros foram obtidos através de fórmulas simples, como indicado a seguir:

ponto de pedido = demanda máxima no tempo de reação; lote fixo = fórmula padrão do lote econômico;

estoque máximo $=$ ponto de pedido mais lote econômico.
Os resultados principais dessa etapa são os melhores parâmetros encontrados de cada modelo de estoque aplicado a cada item, e os respectivos valores dos custos de operação mensais médios. Numericamente temos 300 itens x 4 modelos $=1200$ resultados.

Foram rodadas oito fases do experimento resultantes da combinação dos parâmetros, mostrada na Tabela 3 .

\section{RESULTADOS COMPUTACIONAIS}

A modelagem foi desenvolvida em VBA do Excel e executada num PC com $526 \mathrm{Mb}$ de RAM e Processador Intel de 1,8 Mhz; cada execução completa de cada fase demorou aproximadamente 15 minutos para os 300 itens. A Figura 1, a seguir, apresenta uma comparação entre os quatro modelos de estoque nas três fases do experimento testadas:

Verifica-se o melhor desempenho do modelo Cálculo de Necessidades nas fases 3 a 8, e um desempenho inferior aos de dois modelos reativos nas fases 1 e 2 . A análise mais detalhada dos resultados fornece as razões deste desempenho.

Tabela 3: Fases de testes de simulação.

\begin{tabular}{|c|c|c|c|c|}
\hline FASE & $\begin{array}{c}\text { CUSTO DE } \\
\text { PEDIDO }\end{array}$ & $\begin{array}{c}\text { GUSTO DE } \\
\text { FALTA }\end{array}$ & $\begin{array}{c}\text { TAXA DE } \\
\text { ARMAZENAGEM }\end{array}$ \\
\hline 1 & 80 & 4 & $2 \%$ & Sem \\
\hline 2 & 80 & 10 & $2 \%$ Sem & Sem \\
\hline 3 & 20 & 4 & $2 \%$ Sem \\
\hline 4 & 20 & 10 & $2 \%$ Com \\
\hline 5 & 80 & 4 & $2 \%$ & Com \\
\hline 6 & 80 & 10 & $2 \%$ & Com \\
\hline 7 & 20 & 4 & $2 \%$ & Com \\
\hline 8
\end{tabular}


A Figura 2, a seguir, apresenta os resultados de todos os custos de operação para cada modelo de estoque, obtidos na Fase 1, para cada família de demanda. Os resultados são apresentados em quatro grandes grupos de demanda ordenados por sua complexidade crescente. Dentro de cada um destes grupos, os resultados estão separados em quatro subgrupos, um para cada modelo de estoque testado, ordenados por modelos na ordem RepMaxC, RepBaseC, LotFixC e CalNec. Finalmente, em cada subgrupo os resultados dos itens estão organizados em ordem crescente das famílias, que coincide com a ordem crescente dos desvios padrão combinados com tempos de espera crescentes, utilizados na geração das demandas e nas simulações.

A Figura 2 mostra que os custos de operação dos modelos Cálculo de Necessidades e Reposição da Base, nos casos de demanda constante e constante com sazonalidade, são superiores aos dos modelos de Reposição do Máximo e Lote Fixo.

Como na Fase 1 o valor do Custo de Pedido é alto, é esperado que os modelos cujas regras impliquem em maior número de compras tenham um custo de operação mais alto, que é o caso destes dois modelos quando não se utiliza o recurso de Necessidades Líquidas Mínimas. Comporta- mento semelhante é esperado na Fase 2, confirmado pelos resultados apresentados na Figura 3, onde somente o Custo de Falta é aumentado em relação à Fase 1. A redução do Custo de Pedido na Fase 3 confirma o impacto deste custo no desempenho dos modelos, permitindo que o Cálculo de Necessidades vença em $69 \%$ dos itens. A figura 4 mostra as dispersões referentes a esta fase.

Em todos os gráficos de dispersão pode-se notar um grande aumento na dispersão dos custos dos modelos reativos nos casos de demandas com inclinação (tendência). A mesma observação ressalta a robustez do modelo de Cálculo de Necessidades em relação às diferentes demandas, devidamente tratadas pelos modelos de previsão, apresentando um padrão de dispersão quase constante em todos os ambientes simulados.

A Figura 5 apresenta os resultados da Fase 5 . O efeito do uso de Necessidades Líquidas Mínimas pode ser observado na comparação entre as Fases 1 e 5, onde nesta última este recurso é introduzido, mantendo-se os demais parâmetros da outra fase. Fica claro que a formação de lotes mínimos nos modelos de Reposição da Base e Cálculo de Necessidades, permitindo um número menor de compras, nivela o desempenho destes em relação aos demais modelos reativos nos

Figura 1: Distribuição dos melhores modelos pelo critério de menor custo de operação.

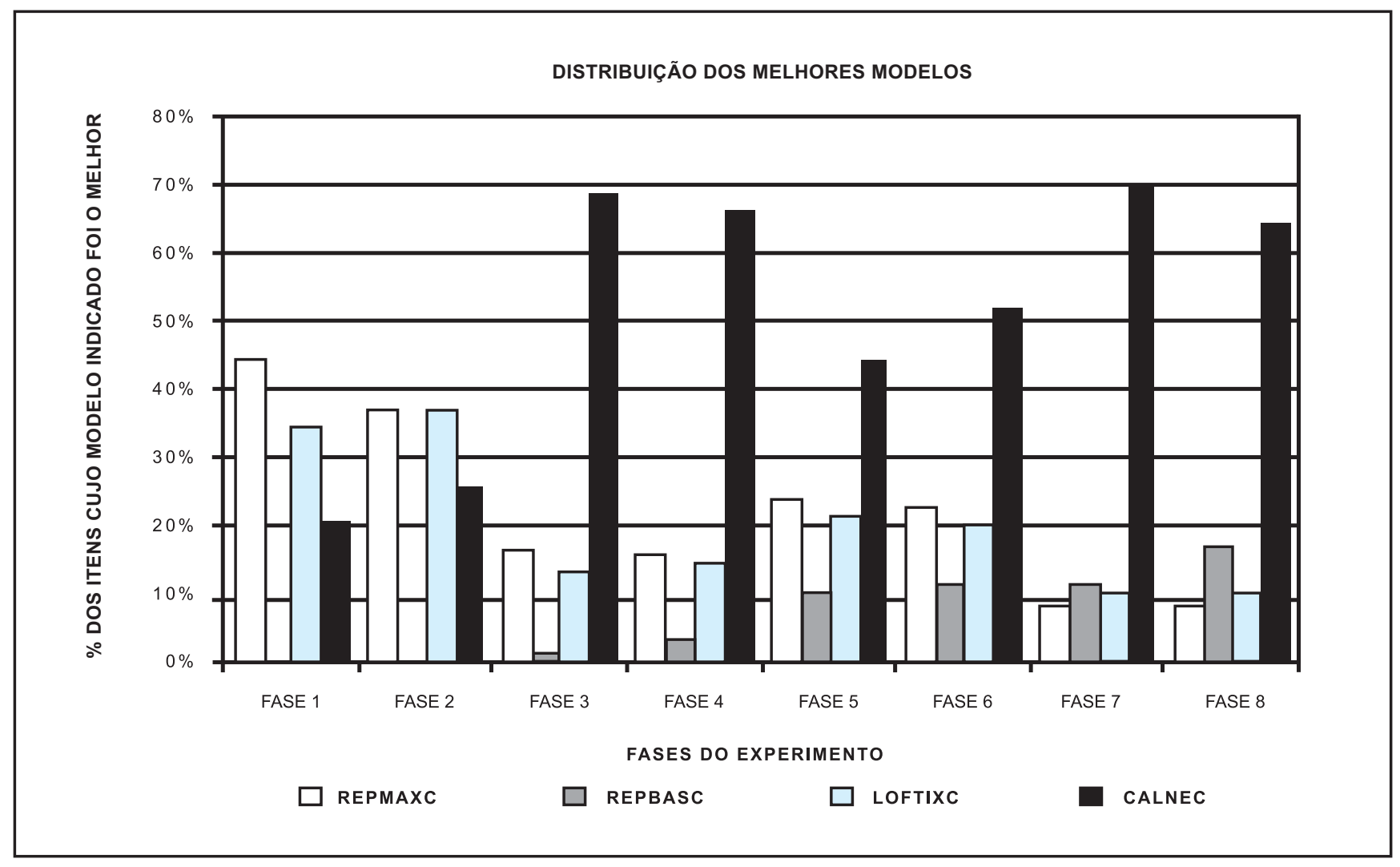


Figura 2: Dispersão dos custos de operação dos itens na Fase 1.

DISPERSÃO DOS CUSTOS DE OPERAÇÃO DOS ITENS FASE 1

CUSTO DE PEDIDO = 80 ; CUSTO DE FALTA $=4$; SEM NECESSIDADES LIQQUIDAS MÍNIMAS

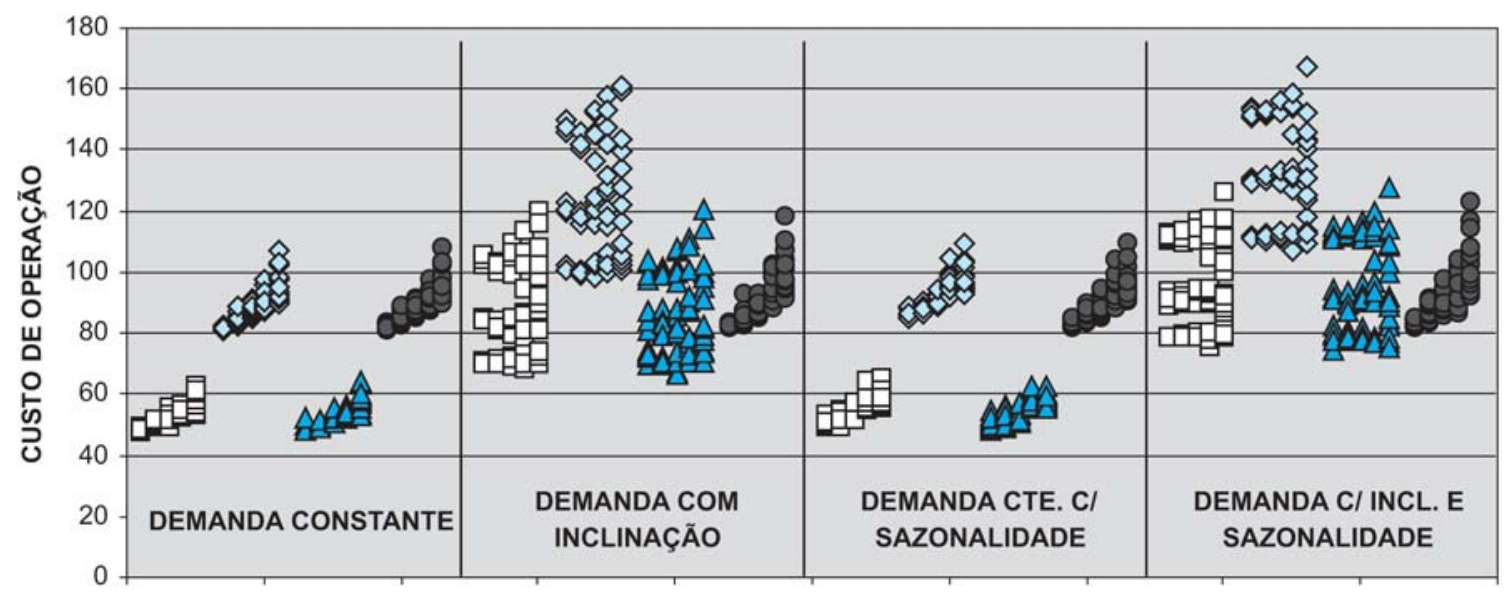

COMPLEXIDADE CRESCENTE DA DEMANDA

口MODELO REPMAXC $\triangle$ MODELOREPBASEC $\triangle$ MODELO LOTFIXOC O MODELO CALNEC

Figura 3: Dispersão dos custos de operação dos itens na Fase 2.

DISPERSÃO DOS CUSTOS DE OPERAÇÃO DOS ITENS FASE 2 CUSTO DE PEDIDO = 80 ; CUSTO DE FALTA $=10$; SEM NECESSIDADES LIQQUIDAS MÍNIMAS

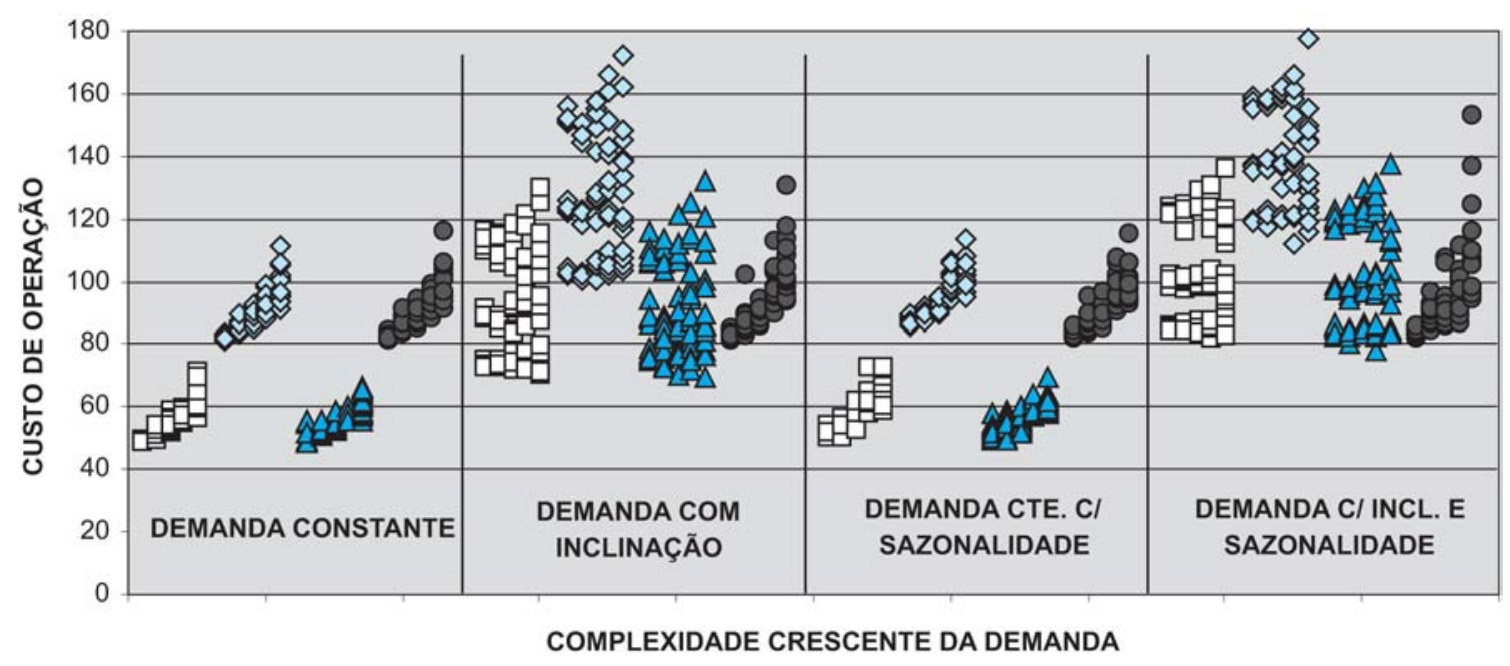

口MODELO REPMAXC $\triangle$ MODELOREPBASEC $\triangle$ MODELO LOTFIXOC OMODELO CALNEC 
Figura 4: Dispersão dos custos de operação dos itens na Fase 3.

DISPERSÃO DOS CUSTOS DE OPERAÇÃO DOS ITENS FASE 3

CUSTO DE PEDIDO = 20 ; CUSTO DE FALTA $=4$; SEM NECESSIDADES LIQQUIDAS MINIMAS

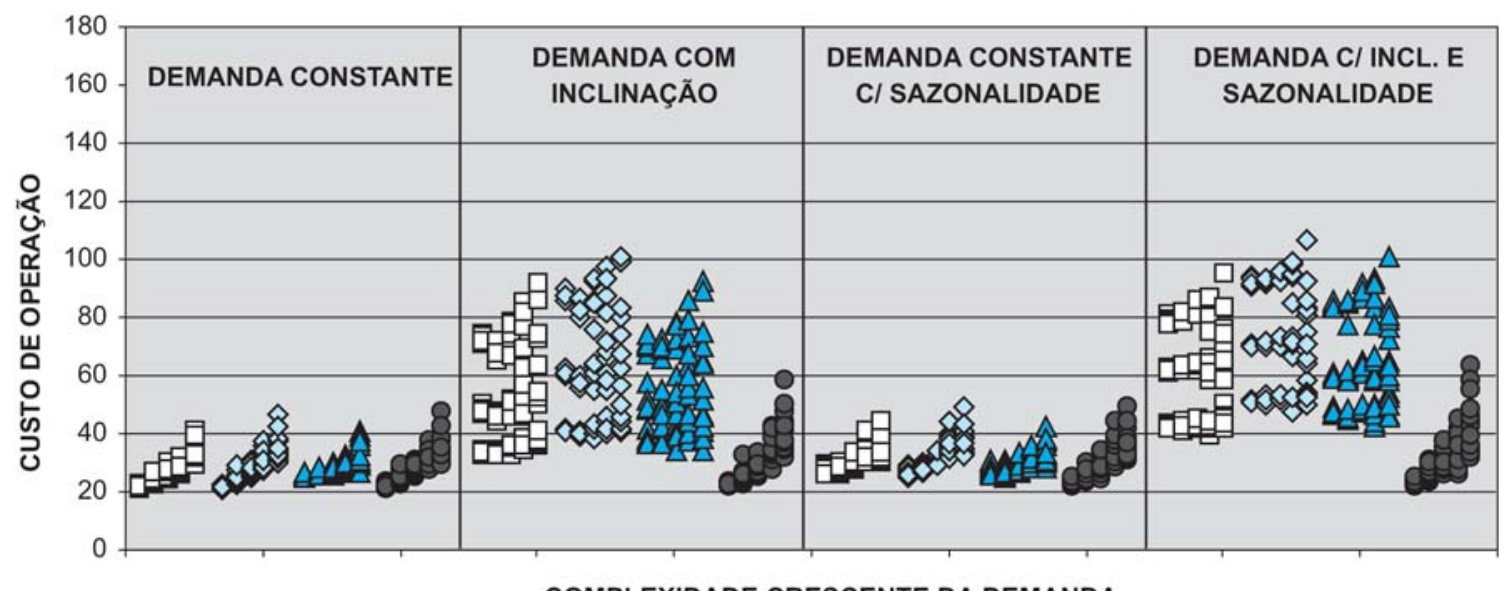

COMPLEXIDADE CRESCENTE DA DEMANDA

口MODELO REPMAXC $\triangle$ MODELO REPBASEC $\triangle$ MODELO LOTFIXOC O MODELO CALNEC

Figura 5. Dispersão dos custos de operação dos itens na Fase 5.

DISPERSÃO DOS CUSTOS DE OPERAÇÃO DOS ITENS FASE 5

CUSTO DE PEDIDO = 80 ; CUSTO DE FALTA $=4$; COM NECESSIDADES LÍQUIDAS MÍNIMAS

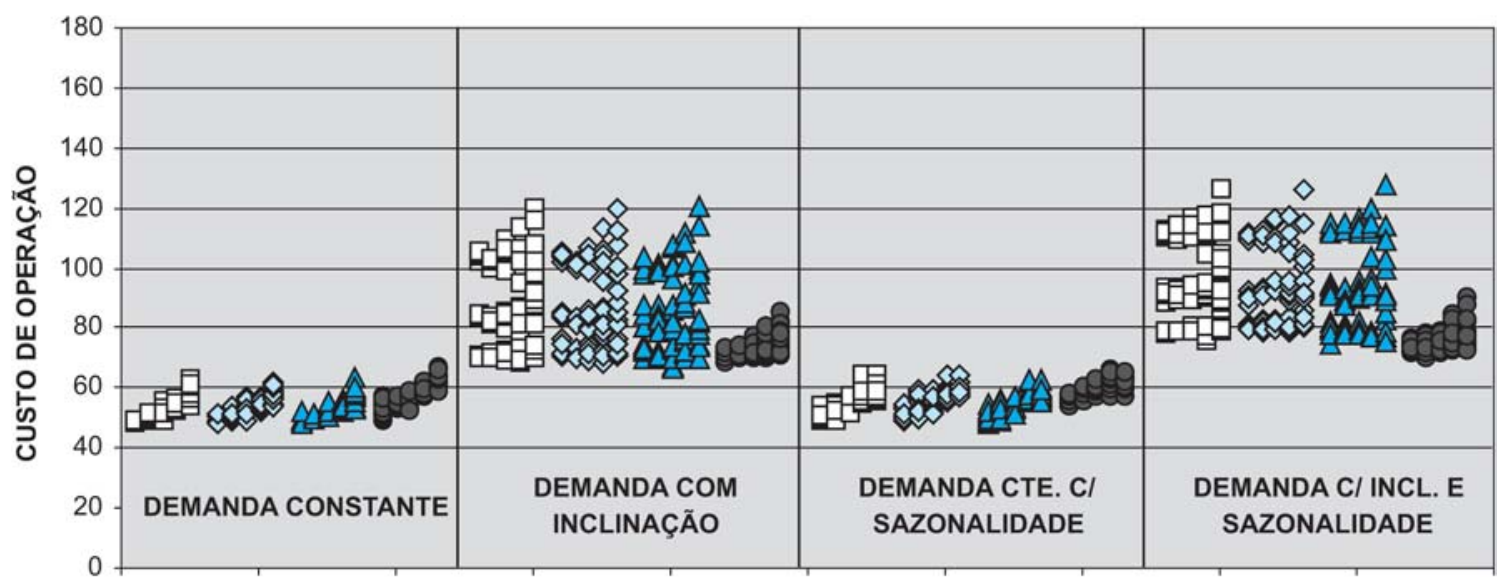

COMPLEXIDADE CRESCENTE DA DEMANDA

$\square$ MODELO REPMAXC $\triangle$ MODELO REPBASEC $\triangle$ MODELO LOTFIXOC O MODELO CALNEC 
ambientes de demanda constante. Para o modelo de Cálculo de Necessidades, o desempenho nos ambientes de demanda com inclinação melhora o suficiente para lhe dar vantagem geral em toda a fase, como pode ser observado na porcentagem de vitórias obtidas (45\%), apresentada na Figura 1.

Para facilitar o entendimento das conclusões a seguir, a Tabela 4 resume o desempenho dos modelos com base nos diferentes custos de pedido (alto e baixo), na consideração ou não de necessidade líquida mínima e na constância ou inclinação da demanda média.

\section{CONCLUSÕES}

Este trabalho efetuou uma análise comparativa do desempenho entre modelos de estoque reativos e ativos, quando submetidos a diferentes demandas. A redução do custo de pedido foi acompanhada por uma substancial melhoria de desempenho do Cálculo de Necessidades e por uma pequena melhoria do modelo Reposição da Base. Isso sugere que a impossibilidade de redução de custo pela compra em lotes maiores penalizou esses dois modelos citados, principalmente o modelo Reposição da Base.

Com Custo de Pedido relativamente baixo e mesmo sem trabalhar com Necessidades Líquidas Mínimas, o Cálculo de Necessidades teve um desempenho superior aos reativos, principalmente quando trabalhou com ambientes de demanda de baixa previsibilidade. Esses resultados estão coerentes com os obtidos por Jacobs e Whybark (1992) embora, a rigor, não se possa comparar efetivamente os resultados pelas diferenças de abordagens e medidas adotadas. No entanto, ao se trabalhar com Custo de Pedido alto os modelos reativos melhoraram muito seus desempenhos relativos e o Cálculo de Necessidades perdeu suas vantagens originais.

A liberação, para o Cálculo de Necessidades e para o Reposição da Base, do uso da Necessidade Líquida Mínima teve, como efeito importante, valores positivos para esse parâmetro como resultado da busca, indicando o interesse nisso em termos absolutos e, em termos relativos, fez com que o Cálculo de Necessidades retomasse sua supremacia em relação aos modelos reativos.

\section{e necessidades líquidas mínimas, mesmo em condições de baixa previsibilidade.}

A existência de demanda com tendência causou, como era de se esperar, um decréscimo acentuado no desempenho relativo dos modelos reativos e um crescimento vigoroso no do Cálculo de Necessidades. A possibilidade de prever, mesmo que com desvios, gera uma vantagem competitiva dificilmente superada pelos modelos reativos que, como a própria designação diz, reagem à demanda.

O resultado sugere um grande interesse no uso de modelos ativos, mesmo em condições de baixa previsibilidade, e o interesse na consideração de quantidades mínimas de aquisição nesse mesmo modelo, pois, mesmo afastando-o de sua origem conceitual coerente com a idéia de JIT, fornece um recurso adicional para seu melhor desempenho, enquanto não forem eliminados os Custos de Pedido.

Tabela 4: Resumo do desempenho dos modelos.

\begin{tabular}{|c|c|c|c|c|c|}
\hline & & \multicolumn{4}{|c|}{ PORGENTAGEM COMO MELHOR MODELO } \\
\hline & & REPMAXC & REPBASEC & LOTFIXC & GALNEG \\
\hline \multirow{2}{*}{ GTOPED } & ALTO & $32 \%$ & $4 \%$ & $28 \%$ & $36 \%$ \\
\hline & BAIXO & $12 \%$ & $9 \%$ & $12 \%$ & $67 \%$ \\
\hline \multirow{2}{*}{ NECLIQMIN } & NÃO & $28 \%$ & $1 \%$ & $25 \%$ & $45 \%$ \\
\hline & SIM & $16 \%$ & $11 \%$ & $16 \%$ & $58 \%$ \\
\hline \multirow{3}{*}{ DEMANDA } & SEM INCLINAÇÃO & $38 \%$ & $13 \%$ & $26 \%$ & $22 \%$ \\
\hline & $\begin{array}{l}\text { COM INGLINAÇÃO } \\
\text { CRESCENTE }\end{array}$ & $20 \%$ & $5 \%$ & $26 \%$ & $49 \%$ \\
\hline & $\begin{array}{l}\text { COM INCLINAÇÃO } \\
\text { DECRESCENTE }\end{array}$ & $8 \%$ & ०\% & $9 \%$ & 83\% \\
\hline
\end{tabular}




\title{
Artigo recebido em 29/06/2006
}

Aprovado para publicação em 02/01/2007

\author{
Referências \\ ARROW, K. A.; HARRIS, T. E.; MARSCHAK, \\ J. Optimal Inventory Policy. Econometrica, \\ 19, p. 250-272, 1951. \\ BROWN, R. G. Decision Rules for Inventory \\ Management. New York: Holt, Rinehart \\ and Winston, 1967. \\ BROWN, R. G. Materials Management \\ Systems. New York: Wiley, 1977. \\ DVORETZKY, A.; KIEFER, J. The Inventory \\ Problems: I. Case of Known Distributions \\ of Demand; II. Case of Unknown \\ Distributions of Demand. Econometrica, \\ 20, p. $187-222$ e p. 450-466p, 1952. \\ EILON, S.; ELMALEH, J. An Evaluation \\ of Alternate Inventory Control Policies. \\ Management Science, v. 16, n. 8, p. \\ 532-548, 1970. \\ HADLEY, G.; WITHIN, T. M. Analysis of \\ Inventory Systems. New Jersey: Prentice \\ Hall, Englewood Cliffs, 1963. \\ FILDES, R.; BEARD, C. Forecasting \\ Systems for Production and Inventory \\ Control. International Journal of Operations \\ \& Production Management, 12, Issues 5, \\ p. 4-27, 1992. \\ FU, M. C.; HEALY, K. J. Techniques for op- \\ timization via simulation: an experimen- \\ tal study on an (s, S) inventory system. \\ IIIE Transaction, 29, p. 191-199, 1997. \\ GARDNER JR, E. S. Evaluating Forecast \\ Performance in an Inventory Control \\ System. Management Science, 36, Iss. 4, \\ p. 490-499, 1990. \\ stochastic inventory models. Computers \\ \& Industrial Engineering, v. 37, n. 1, 2, p. \\ 215-218, 1999. \\ HAX, ARNOLDO; CANDEA, D. Production \\ and Inventory Management. New Jersey: \\ Prentice-Hall, 1984. \\ JACOBS, F. R.; WHYBARK, D. C. A \\ Comparison of Reorder Point and \\ Material Requirements Planning \\ Inventory Control. Logic Decision Sciences, \\ v. 23, n. 2, p. 332-342, 1992. \\ LOPEZ-GARCIA, L.; POSADA-BOLIVAR, \\ A. A simulator that uses Tabu Search \\ to approach the optimal solution to \\ MAKRIDAKIS, S.; S. C. WHEELRIGHT \\ Forecasting-Methods and Applications New \\ York: Wiley, 3. ed, 1998. \\ NADDOR, E. Inventory Systems. New York: \\ Wiley, 1966. \\ ORLICKY, J. Material Requirements Planning. \\ New York: McGraw-Hill, 1995. \\ SIPPER, D. AND BULFIN, R. Production: \\ planning, control and integration. New \\ York: McGraw-Hill, 1998.
}

\section{- Sobre os autores}

\section{Miguel Cezar Santoro}

Professor

Departamento de Engenharia de Produção - Escola Politécnica - USP

End.: Av. Prof. Almeida Prado, 128 - Cidade Universitária - 05508-900 - São Paulo - SP

Tel.: (11) 3091-5363

E-mail: santoro@usp.br

\section{Gilberto Freire}

Mestre em Engenharia de Produção

Departamento de Engenharia de Produção - Escola Politécnica - USP

End.: Av. Prof. Almeida Prado, 128 - Cidade Universitária - 05508-900 - São Paulo - SP

Tel.: (11) 3091-5363

E-mail: gfreire@usp.br 